\title{
Estudio preliminar del esqueleto postcraneal del Muscardinus cyclopeus Agustí, Moyà-Solà \& Pons-Moyà, 1982 (Mammalia, Rodentia, Myoxidae)
}

\author{
Preliminary study of the postcranial skeleton of Muscardinus \\ cyclopeus Agustí, Moyà-Solà \& Pons-Moyà, 1982 (Mammalia, \\ Rodentia, Myoxidae)
}

\author{
J. Quintana Cardona ${ }^{1,2}$ \\ 1 Institut Català de Paleontologia Miquel Crusafont (ICP). Edificio ICP. Campus de la Universidad Autónoma de Barcelona \\ 08193 Cerdanyola del Vallés (Barcelona) \\ 2 Gustau Mas, 79-1er 07760 Ciutadella de Menorca (Illes Balears). Email: picoguevo@hotmail.com
}

\section{RESUMEN}

Se describe, por primera vez, parte del esqueleto del muscardino gigante del Plioceno de Menorca, procedente del yacimiento 3 de Punta Nati, situado al noroeste del término municipal de Ciutadella de Menorca (Islas Baleares, Mediterráneo occidental). A partir de los parámetros óseos se ha podido constatar que Muscardinus cyclopeus muestra, de media, un tamaño un 69\% superior al de Muscardinus avellanarius (Linnaeus, 1758). Entre las principales características óseas que diferencian $M$. cyclopeus de $M$. avellanarius cabe destacar, entre otras, el diámetro proporcionalmente más ancho de la epífisis proximal de la ulna, el olecranon proporcionalmente más corto, la fóvea de la epífisis proximal del radio con una concavidad menos marcada, el ilium proporcionalmente más corto, los epicóndilos de la epífisis distal del fémur más simétricos, el epicóndilo lateral proporcionalmente más alto en sentido dorso-ventral, con la superficie ventral muy aplanada y la tibia proporcionalmente más estrecha en sentido transversal. Al tratarse de una especie evolucionada en un contexto insular, resulta difícil dilucidar cuales son caracteres primitivos y caracteres derivados.

Palabras clave: Lirón Gigante; Evolución Insular; Incremento de Tamaño; Faunas Vertebradas Endémicas; Yacimientos Cársticos.

\section{ABSTRACT}

For the first time, the partial skeleton of the Pliocene Muscardinus cyclopeus is described from Punta Nati-3, located in the northwest of the municipality of Ciutadella de Menorca (Balearic Islands, western Mediterranean). Based on bone parameters it is shown that the size of Muscardinus cyclopeus is on overage $69 \%$ higher than that of Muscardinus avellanarius. The main features that distinguish $M$. cyclopeus from $M$. avellanarius are, among others: the relatively wider diameter of the proximal epiphysis of the ulna; the relatively shorter olecranon; the less marked concavity in the fovea of the proximal epiphysis of the radius; the relatively shorter ilium;

Recibido el 10 de diciembre de 2013 / Aceptado el 4 de junio de 2014 / Publicado online el 2 de marzo de 2015

Citation / Cómo citar este artículo: Quintana Cardona, J. (2015). Estudio preliminar del esqueleto postcraneal del Muscardinus cyclopeus Agustí, Moyà-Solà \& Pons-Moyà, 1982 (Mammalia, Rodentia, Myoxidae). Estudios Geológicos 71(1): e022. http://dx.doi. org/10.3989/egeol.41618.305.

Copyright: () 2015 CSIC. This is an open-access article distributed under the terms of the Creative Commons Attribution-Non Commercial (by-nc) Spain 3.0 License. 
the more symmetrical epicondyles of the distal femoral epiphysis; the relatively higher lateral epicondyle dorsoventrally, with a very flat ventral surface; and the relatively narrower tibia transversely. As a species evolved in an insular context, it is still very difficult to discern which of these characters are primitive and which are derived.

Keywords: Giant Dormouse; Insular Evolution; Size Increase; Vertebrate Endemic Faunas; Karstic Sites.

\section{Introducción}

Las faunas de mamíferos terrestres (no voladores) post-messinienses de las islas Baleares se caracterizan, entre otras cosas, por el reducido número de especies de roedores. Sin lugar a dudas, es en los yacimientos más antiguos del Plioceno inferior (en los que se han descrito las denominadas "faunas tempranas" (sensu Quintana \& Moncunill-Solé, 2014a), donde existe una mayor diversidad de roedores. Considerando las Baleares en su conjunto, los lirones son el grupo de roedores que dominan sobre el resto de familias (cricétidos, múridos y gerbílidos) en los yacimientos más antiguos y los que perduran hasta la llegada de los primeros humanos a las islas durante el Holoceno.

Del conjunto de islas que forman las Baleares, Menorca es la que muestra, durante el Neógeno, una historia biogegráfico-mastológica más compleja (Quintana \& Moncunill-Solé, 2014a: fig. 11). Existen, desde el Plioceno inferior hasta el holoceno, dos grupos faunísticos diferentes. El más antiguo, situado en el plioceno superior, está caracterizado, entre otros, por la tortuga gigante de Menorca (Cheirogaster gymnesica [Bate, 1914]), el conejo gigante de Menorca (Nuralagus rex Quintana, Köhler \& Moyà, 2011) y el muscardino gigante de Menorca (Muscardinus cyclopeus Agustí, Moyà-Solà \& PonsMoyà, 1982) (fig. 1).

El segundo grupo faunístico habría colonizado Menorca desde Mallorca, posiblemente en el Pleistoceno inferior, una vez desaparecidos casi todos los taxones presentes en el primer grupo faunístico (entre los vertebrados terrestres no voladores, la lagartija balear, Podarcis lilfordi (Günter, 1874) y el sapo partero gimnésico, Alytes muletensis (Sanchiz \& Adrover, 1977) habrían sido los únicos taxones supervivientes). Este segundo grupo faunístico estaría formado por un bóvido del género Myotragus Bate, 1909, un insectívoro del género Nesiotites Bate, 1944 y un lirón del género Hypnomys Bate, 1918.

En la descripción original de M. cyclopeus no se cita, describe o figura ninguna parte esquelética, a excepción, obviamente, de los dientes (Agustí et al.,
1982). La localización (en marzo de 1988) de un nuevo yacimiento (Punta Nati-3) con restos relativamente abundantes de M. cyclopeus (Quintana, 1998) permite ahora abordar el estudio preliminar de algunas de las partes esqueléticas de este lirón.

\section{Contexto geográfico y geológico}

La totalidad de los restos de M. cyclopeus fueron recuperados del yacimiento de Punta Nati-3, en el extremo noroccidental del término municipal de Ciutadella de Menorca (fig. 2). Esta zona se caracteriza, entre otras cosas, por la presencia de numerosos yacimientos de extensión bastante limitada con restos de vertebrados, cuyo origen se relaciona con uno o varios sistemas cársticos desarrollados tanto sobre las dolomías jurásicas como en los

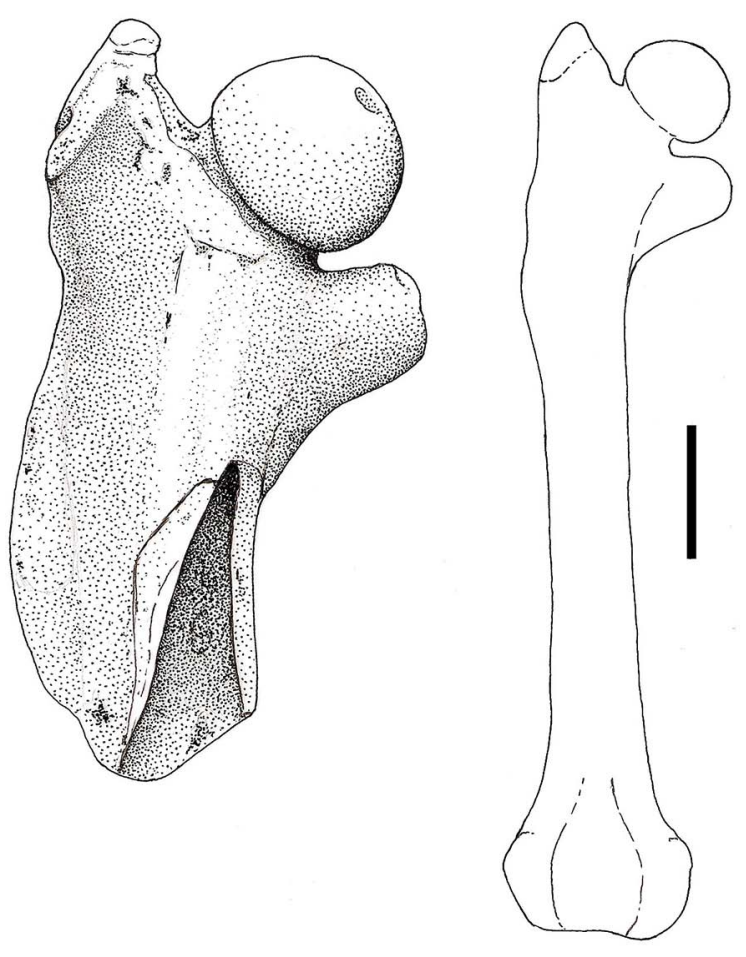

Fig. 1.-Diferencias de tamaño entre la epífisis proximal derecha de M. cyclopeus (CBQ-4165) (izquierda) y el fémur de $M$. avellanarius (derecha). Escala gráfica: $2 \mathrm{~mm}$. 

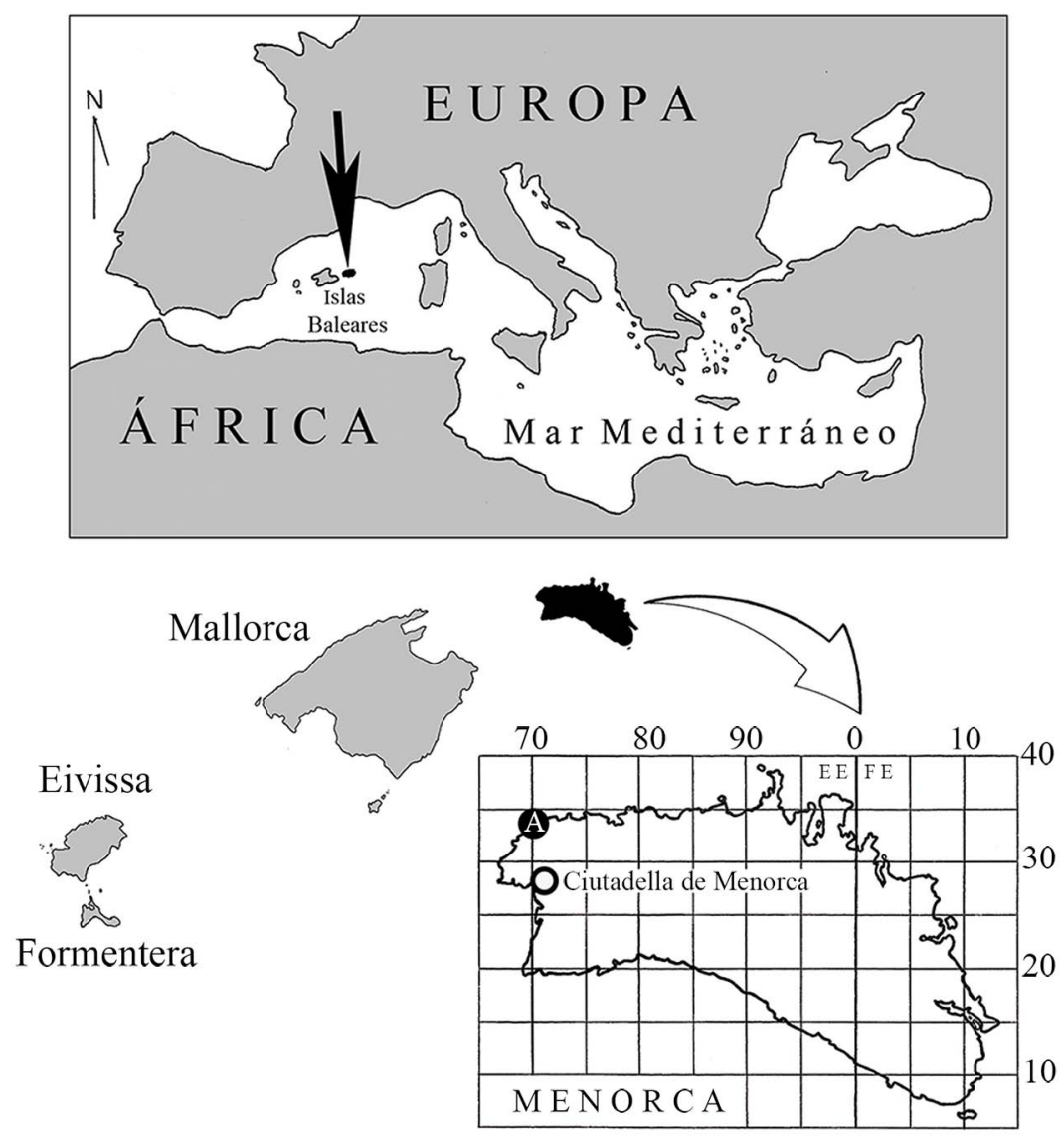

Fig. 2.-Situación geográfica del yacimiento de Punta Nati-3 (punto A).

sedimentos miocénicos existentes en la zona. Los limos que forman el yacimiento de Punta Nati-3 descansan directamente sobre el basamento dolomítico jurásico. Son fuertemente rojizos y muy duros, al estar cementados por carbonato de calcio (Quintana, 2005: fig. 1C y fig. 2).

El yacimiento de Punta Nati-3 incluye, además de $M$. cyclopeus, un lepórido (N. rex), un quiróptero (Rhinolophus euryale Blasius, 1853) (Sevilla et al., 2013), varios reptiles (Ch. gymnesica y un lacértido indeterminado) y varias aves (Tyto balearica Mourer-Chauviré, Alcover, Moyà \& Pons, 1980 y Pterodromoides minoricensis Seguí, Quintana, Fornós \& Alcover, 2001) (Seguí, 1998; Quintana, 2005).

\section{Metodología de trabajo}

La separación de los restos óseos de la roca matriz se realizó sumergiendo, repetidas veces, las muestras en una solución de ácido acético y agua en una concentración del 10\%. Una vez separados los fósiles de la roca matriz, se sumergieron (durante varias horas) en agua con el fin de eliminar cualquier resto de ácido o sal básica. Los huesos ya secos fueron consolidados con una solución de Paraloid B-67 en acetona en una concentración del $10 \%$.

Los huesos recuperados se conservan, de forma individual, en tubos de cristal ( $\mathrm{L}=40 \mathrm{~mm} ; \varnothing=10 \mathrm{~mm})$ y actualmente se encuentran depositados en la colección de historia natural del autor (acrónimo CBQ: Colección Bep Quintana, Ciutadella de Menorca. Islas Baleares, España). Todos los ejemplares se conservan en unas condiciones seguras y estables, y están disponibles a cualquier investigador, sin ningún tipo de restricción. M. cyclopeus ha sido comparado con dos esqueletos de Muscardinus avellanarius (Linnaeus, 1758) procedentes del National Museums of Scotland (Department of Natural Sciences) con los números de registro 360/93 y 364/93. La comparación no ha sido posible en el caso de los astrágalos, 
ausentes en los esqueletos de $M$. avellanarius. Todas las medidas (tabla 1 y 2) han sido realizadas a partir de fotografías, utilizando un binocular con micrómetro incorporado, según los parámetros expresados en la fig. 3.

\section{Objetivos}

M. cyclopeus es, sin duda, uno de los mamíferos más desconocidos del Neógeno balear. Tan solo muy recientemente, $M$. cyclopeus ha sido incluido en un estudio en el que se relaciona la masa corporal de algunos roedores insulares con la superficie de las islas (Moncunill-Solé et al., 2014).
En este trabajo se describe y figura parte del esqueleto de M. cyclopeus y se compara con el de M. avellanarius, a fin de analizar si las diferencias observadas pueden relacionarse o no con la evolución en una isla.

\section{Paleontología sistemática}

Orden RODENTIA Bowdich, 1821

Familia MYOXIDAE (Gray, 1821)

Subfamilia MYOXINAE (Gray, 1821)

Género Muscardinus Kaup, 1829

Muscardinus cyclopeus Agustí, Moyà-Solà \& PonsMoyà, 1982

Tabla 1.-Medidas, en $\mathbf{m m}$, de la extremidad anterior en $\boldsymbol{M}$. cyclopeus y $\boldsymbol{M}$. avellanarius. $\mathrm{n}$ : número de individuos; SD: desviación estándar

\begin{tabular}{|c|c|c|c|c|c|c|c|c|}
\hline \multicolumn{9}{|c|}{ EXTREMIDAD ANTERIOR } \\
\hline \multicolumn{9}{|c|}{ HÚMERO (epífisis proximal) } \\
\hline & \multicolumn{4}{|c|}{ Diámetro dorso-ventral } & \multicolumn{4}{|c|}{ Diámetro transversal } \\
\hline Especie & $\mathrm{n}$ & Máx.-Mín & Media & SD & $\mathrm{n}$ & Máx.-Mín & Media & SD \\
\hline M. cyclopeus & 10 & $3,72-3,22$ & 3,48 & 0,1843 & 10 & $4,41-3,94$ & 4,07 & 0,1353 \\
\hline M. avellanarius & 2 & $2,14-2,02$ & 2,08 & 0,0848 & 2 & $2,71-2,42$ & 2,56 & 0,4879 \\
\hline \multicolumn{9}{|c|}{ HÚMERO (epífisis distal) } \\
\hline & \multicolumn{4}{|c|}{ Diámetro transversal } & \multicolumn{4}{|c|}{ Diámetro transversal sup. articulación } \\
\hline Especie & $\mathrm{n}$ & Máx.-Mín & Media & SD & $\mathrm{n}$ & Máx.-Mín & Media & SD \\
\hline M. cyclopeus & 5 & $5,35-5,14$ & 5,25 & 0,0944 & 5 & $3,50-3,31$ & 3,41 & 0,0770 \\
\hline M. avellanarius & 2 & $3,29-3,23$ & 3,26 & 0,6151 & 2 & $2,08-1,96$ & 2,02 & 0,8131 \\
\hline \multicolumn{9}{|c|}{ HÚMERO (epífisis distal) } \\
\hline & \multicolumn{4}{|c|}{ Diámetro dorso-ventral sup. art. } & \multicolumn{4}{|c|}{ Diámetro cráneo-caudal sup. art. } \\
\hline Especie & $\mathrm{n}$ & Máx.-Mín & Media & SD & $\mathrm{n}$ & Máx.-Mín & Media & SD \\
\hline M. cyclopeus & 5 & $1,56-1,49$ & 1,53 & 0,0383 & 5 & $1,72-1,54$ & 1,63 & 0,0746 \\
\hline M. avellanarius & 2 & $1,11-1,04$ & 1,07 & 0,1555 & 2 & $0,97-0,95$ & 0,96 & 0,0494 \\
\hline \multicolumn{9}{|c|}{ RADIO (epífisi proximal) } \\
\hline & \multicolumn{4}{|c|}{ Diámetro cráneo-caudal } & \multicolumn{4}{|c|}{ Diámetro transversal } \\
\hline Especie & $\mathrm{n}$ & Máx.-Mín & Media & SD & $\mathrm{n}$ & Máx.-Mín & Media & SD \\
\hline M. cyclopeus & 9 & $1,97-1,58$ & 1,67 & 0,1236 & 9 & $2,42-2,12$ & 2,20 & 0,1007 \\
\hline M. avellanarius & 2 & $1,07-1,02$ & 1,04 & 0,0848 & 2 & $1,40-1,33$ & 1,36 & 0,0494 \\
\hline \multicolumn{9}{|c|}{ ULNA (epífisis proximal) } \\
\hline & \multicolumn{4}{|c|}{ Diámetro dorso-ventral incisura troclear } & \multicolumn{4}{|c|}{ Profundidad cráneo-caudal incisura troclear } \\
\hline Especie & $\mathrm{n}$ & Máx.-Mín & Media & SD & $\mathrm{n}$ & Máx.-Mín & Media & SD \\
\hline M. cyclopeus & 11 & $2,21-1,99$ & 2,09 & 0,0790 & 11 & $1,00-0,86$ & 0,92 & 0,0459 \\
\hline M. avellanarius & 2 & $1,22-1,09$ & 1,15 & 0,0777 & 2 & 0,56 & 0,56 & - \\
\hline \multicolumn{9}{|c|}{ ULNA (epífisis proximal) } \\
\hline & \multicolumn{4}{|c|}{ Altura olecranon } & \multicolumn{4}{|c|}{ Diámetro transversal } \\
\hline Especie & $\mathrm{n}$ & Máx.-Mín & Media & SD & $\mathrm{n}$ & Máx.-Mín & Media & SD \\
\hline M. cyclopeus & 11 & $2,28-1,60$ & 2,09 & 0,1979 & 10 & $3,10-2,44$ & 2,90 & 0,1906 \\
\hline M. avellanarius & 2 & $1,50-1,39$ & 1,44 & 0,6646 & 2 & $1,41-1,14$ & 1,27 & 0,0141 \\
\hline
\end{tabular}


Tabla 2.-Medidas, en $\mathrm{mm}$, de la extremidad posterior en $\boldsymbol{M}$. cyclopeus y $\boldsymbol{M}$. avellanarius. $\mathrm{n}$ : número de individuos; SD:desviación estándar

\begin{tabular}{|c|c|c|c|c|c|c|c|c|}
\hline \multicolumn{9}{|c|}{ EXTREMIDAD POSTERIOR } \\
\hline \multicolumn{9}{|c|}{ FÉMUR (epífisis proximal) } \\
\hline & \multicolumn{4}{|c|}{ Distancia entre trocánters } & \multicolumn{4}{|c|}{ Diámetro transversal caput femoris } \\
\hline Especie & $\mathrm{n}$ & Máx.-Mín & Media & SD & $\mathrm{n}$ & Máx.-Mín & Media & SD \\
\hline M. cyclopeus & 4 & $6,40-5,55$ & 6,04 & 0,3561 & 4 & $2,81-2,64$ & 2,75 & 0,0785 \\
\hline \multirow[t]{3}{*}{ M. avellanarius } & 1 & - & 3,72 & - & 2 & $1,57-1,54$ & 1,55 & 0,3040 \\
\hline & \multicolumn{4}{|c|}{ FÉMUR(epífisis proximal) } & \multicolumn{4}{|c|}{ FÉMUR (epífisi distal) } \\
\hline & \multicolumn{4}{|c|}{ Diámetro cráneo-caudal caput femoris } & \multicolumn{4}{|c|}{ Diámetro transversal } \\
\hline Especie & $\mathrm{n}$ & Máx.-Mín & Media & SD & $\mathrm{n}$ & Máx.-Mín & Media & SD \\
\hline M. cyclopeus & 4 & $2,07-1,78$ & 1,93 & 0,1400 & 7 & $5,18-4,80$ & 4,93 & 0,1593 \\
\hline M. avellanarius & 2 & $1,10-1,07$ & 1,08 & 0,3111 & 2 & $2,90-2,70$ & 2,80 & 0,1414 \\
\hline \multicolumn{9}{|c|}{ TIBIA (epífisis distal) } \\
\hline & \multicolumn{4}{|c|}{ Diámetro cráneo-caudal } & \multicolumn{4}{|c|}{ Diámetro transversal } \\
\hline Especie & $\mathrm{n}$ & Máx.-Mín & Media & SD & $\mathrm{n}$ & Máx.-Mín & Media & SD \\
\hline M. cyclopeus & 2 & $2,24-1,90$ & 2,07 & 0,2404 & 2 & $3,95-3,55$ & 3,75 & 0,2828 \\
\hline M. avellanarius & 2 & $1,35-1,23$ & 1,29 & 0,1979 & 2 & $2,33-2,24$ & 2,28 & 0,7778 \\
\hline \multicolumn{9}{|c|}{ CALCÁNEO } \\
\hline & \multicolumn{4}{|c|}{ Diámetro cráneo-caudal } & \multicolumn{4}{|c|}{ Diámetro transversal } \\
\hline Especie & $\mathrm{n}$ & Máx.-Mín & Media & SD & $\mathrm{n}$ & Máx.-Mín & Media & SD \\
\hline M. cyclopeus & 8 & $5,79-5,29$ & 5,45 & 0,1645 & 16 & $3,10-2,60$ & 2,77 & 0,1306 \\
\hline M. avellanarius & 1 & - & 2,93 & - & 1 & - & 1,70 & - \\
\hline \multicolumn{9}{|c|}{ ASTRÁGALO } \\
\hline & \multicolumn{4}{|c|}{ Diámetro cráneo-caudal } & \multicolumn{4}{|c|}{ Diámetro transversal } \\
\hline Especie & $\mathrm{n}$ & Máx.-Mín & Media & SD & $\mathrm{n}$ & Máx.-Mín & Media & SD \\
\hline M. cyclopeus & 7 & $4,50-3,71$ & 4,05 & 0,2692 & 7 & $1,96-1,58$ & 1,83 & 0,1428 \\
\hline
\end{tabular}

\section{Extremidad anterior}

Húmero

Material. CBQ-4187, CBQ-4190 (epífisis proximales derechas), CBQ-4188, CBQ-4189, CBQ-4191, CBQ-4192, CBQ-4193, CBQ-4194, CBQ-4195, CBQ-4259, CBQ-4260, CBQ-4343, (epífisis proximales izquierdas), CBQ-4181 (epífisis distal derecha), CBQ-4180, CBQ-4182, CBQ-4185, CBQ-4186, (epífisis distales izquierdas).

Descripción. En la epífisis proximal de M. cyclopeus, el sulco intertubercular forma, comparado con $M$. avellanarius, una concavidad más estrecha y profunda en sentido transversal (fig. 4C y 4D). En la epífisis distal, la cresta lateral de la troclea muestra un perfil más agudo que en $M$. avellanarius; la cresta medial de $M$. cyclopeus también muestra un perfil algo más agudo, dado que la mitad medial de la superficie de la troclea muestra, por su cara ventral, un perfil prácticamente recto, mientras que en $M$. avellanarius es ligeramente convexa. El epicóndilo medial de M. cyclopeus muestra un perfil redondeado en forma de "U" abierta, mientras que en $M$. avellanarius es algo más alargado en sentido cráneo-caudal y con el margen distal más agudo (fig. 4A y 4B, tabla 1).

Comentario. Cabe destacar que de los doce extremos proximales de húmero que se conservan, nueve de ellos (75\%) están sin epifisar. Dado que las diferencias morfométricas entre las epífisis soldadas y sin epifisar son pequeñas, es probable que tal característica esté relacionada con el alargamiento del ciclo de vida de M. cyclopeus y el retraso de su madurez, tal como se ha observado en el género Hypnomys a partir del estudio de la histología ósea (García-Martínez et al., 2011).

\section{Radio}

Material. CBQ-4210, CBQ-4211, CBQ-4212, CBQ-4215, CBQ-4216 (epífisis proximales izquierdas), CBQ-4213, CBQ-4214, CBQ-4217, CBQ-4218, CBQ-4219 (epífisis proximales derechas). 


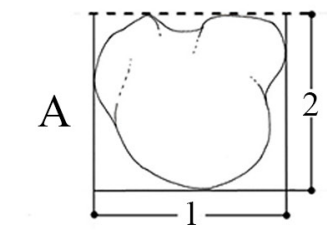

$\mathrm{B}$
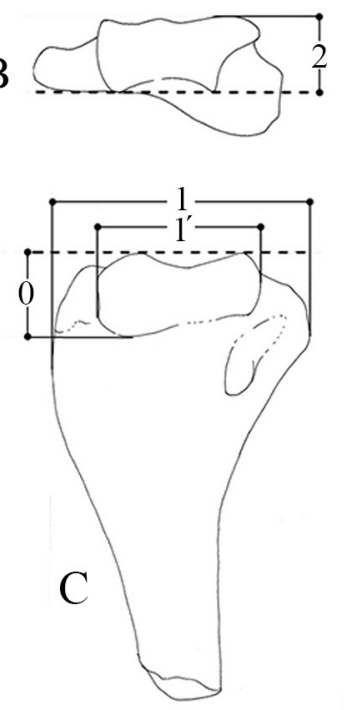
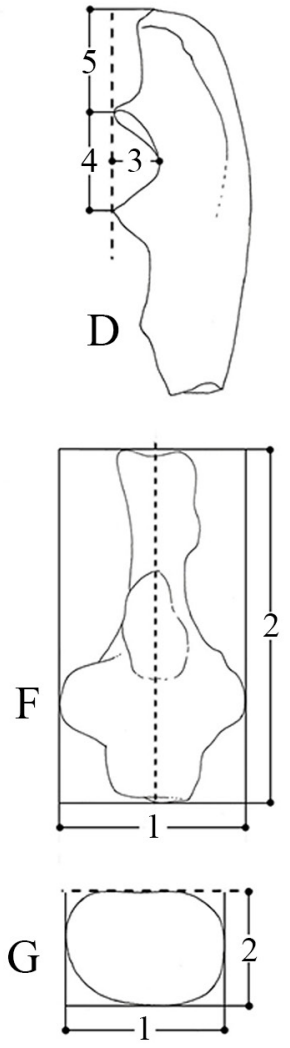
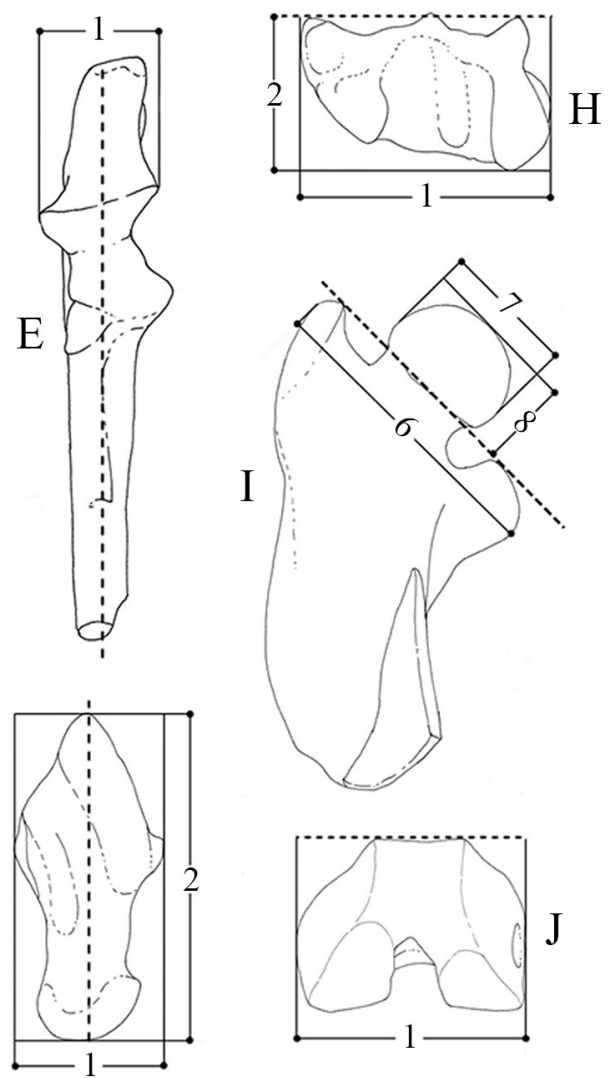

$\mathrm{K}$

Fig. 3.-Medidas osteológicas. A: epífisis proximal del húmero (1: diámetro transversal; 2: diámetro dorso-ventral); B: epífisis distal del húmero, vista caudal (2: diámetro dorso-ventral); C: epífisis distal del húmero, vista ventral (0: diámetro cráneo-caudal de la superficie de articulación; 1: diámetro transversal; 1': diámetro transversal de la superficie de articulación); D: epífisis proximal de la ulna, vista medial (3: profundidad de la tróclea; 4: diámetro dorso-ventral de la tróclea; 5: altura del olecranon); E: epífisis proximal de la ulna, vista craneal (1: diámetro transversal); F: calcáneo, vista dorsal (1: diámetro transversal; 2: diámetro cráneo-caudal); G: epífisis proximal del radio, vista dorsal (1: diámetro transversal; 2: diámetro cráneo-caudal); H: epífisis distal de la tibia, vista ventral (1: diámetro transversal; 2: diámetro cráneo-caudal); I: epífisis proximal del fémur, vista dorsal (6: distancia entre el trocánter mayor y menor; 7: diámetro transversal de la cabeza del fémur; 8: diámetro cráneo-caudal de la cabeza del fémur); J: epífisis distal del fémur, vista craneal (1: diámetro transversal); K: astrágalo, vista dorsal (1: diámetro transversal; 2: diámetro cráneo-caudal). Las líneas discontinuas son las guías a partir de las cuales se definen los parámetros medidos.

Descripción. Visto por su cara craneal, el margen medial de la epífisis proximal del radio de M. cyclopeus es más bajo (en sentido dorso-ventral) que el de $M$. avellanarius, de forma que la fovea de $M$. cyclopeus muestra una concavidad menos marcada en su mitad medial. Vista por su cara dorsal, la fovea de la epífisis proximal del radio de $M$. cyclopeus muestra un contorno redondeado algo achatado, con el margen caudal muy aplanado, mientras que en $M$. avellanarius el contorno es algo más agudo en el margen medial y más sinuoso (convexo) en el margen caudal (figs. $4 \mathrm{~K}-4 \mathrm{~N}$, tabla 1 ).

\section{Ulna}

Material. CBQ-4199, CBQ-4202, CBQ-4203, CBQ-4204, CBQ-4207 (epífisis proximales izquierdas), CBQ-4197, CBQ-4198, CBQ-4200, CBQ4201, CBQ-4205, CBQ-4206, CBQ-4208, CBQ-420 (epífisis proximales derechas).

Descripción. Las diferencias entre la epífisis proximal de M. cyclopeus y M. avellanarius son pequeñas. Vista por su cara medial, los márgenes dorsal y ventral de la incisura troclear de $M$. cyclopeus están, en relación al plano horizontal, más inclinados hacia la zona ventral que en $M$. avellanarius. La faceta lateral 

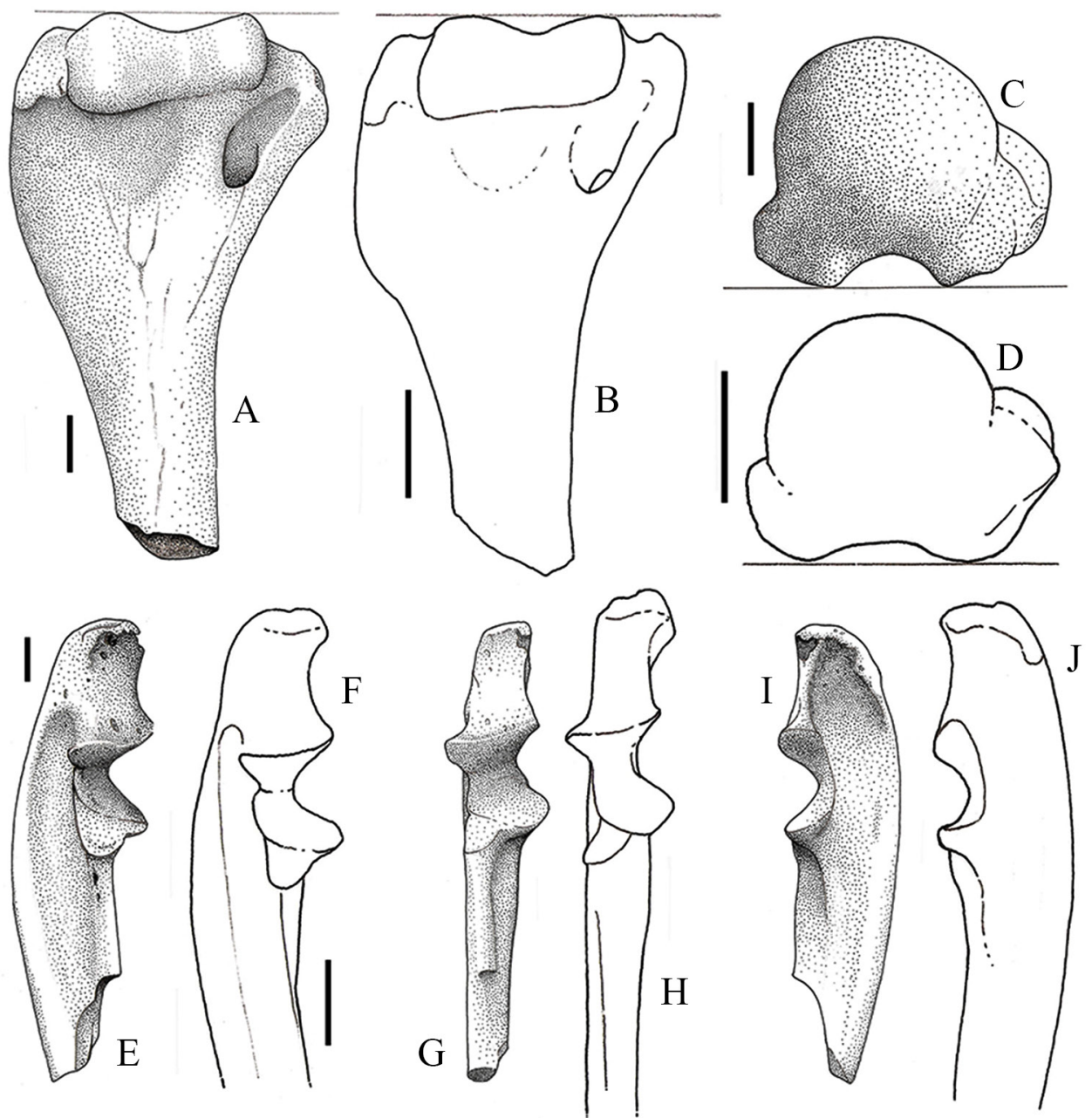

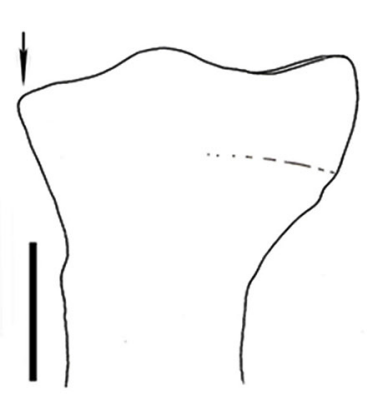

$\mathrm{K}$

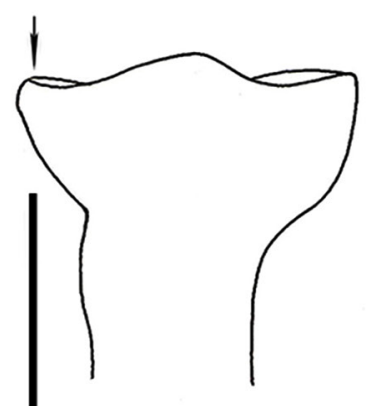

$\mathrm{L}$

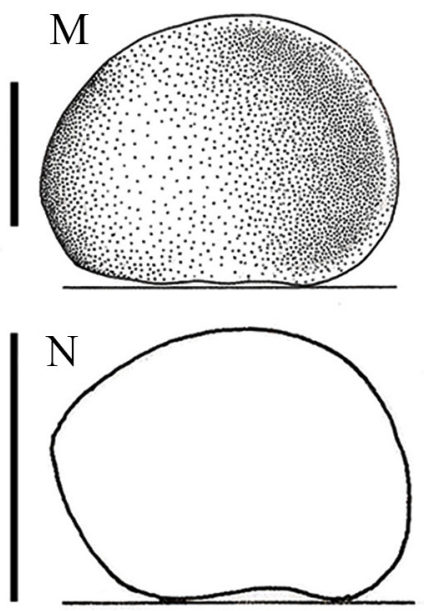

Fig. 4.-Extremidad anterior de M. cyclopeus (A: epífisis distal del húmero izquierdo [CBQ-4182], vista ventral. C: epífisis proximal del húmero izquierdo [CBQ-4259], vista craneal. Epífisis proximal de la ulna derecha [CBQ-4198]: vista lateral [E], craneal [G] y medial [l]. Epífisis proximal del radio izquierdo [CBQ-4210]: vista craneal [K] y dorsal [M]) Extremidad anterior de M. avellanarius (360/93)

(B:epífisis distal del húmero izquierdo, vista ventral. D: epífisis proximal del húmero izquierdo, vista craneal. Epífisis proximal de la ulna derecha, vista lateral $[\mathrm{F}]$, craneal $[\mathrm{H}]$ y medial $[\mathrm{J}]$. Epífisis proximal del radio izquierdo, vista craneal $[\mathrm{L}]$ y dorsal $[\mathrm{N}])$. Escala: $1 \mathrm{~mm}$. 
para la articulación del húmero de $M$. cyclopeus también está más inclinada hacia la zona ventral que en M. avellanarius. El olecranon de M. cyclopeus es proporcionalmente más corto en sentido dorso-ventral (figs. 4E-4J, tabla 1).

\section{Extremidad posterior}

\section{Pelvis}

Material. CBQ-4220 (fragmento de pelvis izquierda), CBQ-4344, CBQ-4345, CBQ-4346, CBQ4347 (fragmentos de pelvis derechas).

Descripción. El ilium de M. cyclopeus presenta un aspecto más robusto, al ser proporcionalmente más corto en sentido cráneo-caudal y más ancho en sentido medio-lateral que M. avellanarius (fig. 5A y 5B).

\section{Fémur}

Material. CBQ-4166, CBQ-4168 (epífisis proximales izquierdas), CBQ-4163, CBQ-4164, CBQ4165, CBQ-4167 (epífisis proximales derechas), CBQ-4169, CBQ-4171, CBQ-4173, CBQ-4174 (epífisis distales izquierdas), CBQ-4170, CBQ-4172, CBQ-4175, CBQ-4176, CBQ-4177, CBQ-4178, CBQ-4179 (epífisis distales derechas).

Descripción. Vista por su cara caudal (fig. 5C y 5D), la zona comprendida entre la cabeza del fémur y el trocánter mayor de $M$. cyclopeus presenta un aspecto más robusto, al ser transversalmente más corta y dorso-ventralmente más ancha que M. avellanarius. Caudalmente, la cabeza del fémur de M. cyclopeus muestra un perfil más redondeado que el de $M$. avellanarius. El margen lateral del trocánter mayor de M. cyclopeus muestra, visto por su cara caudal, un perfil sinuoso, mientras que en M. avellanarius el perfil es más redondeado. El trocánter mayor de M. cyclopeus es proporcionalmente más estrecho en sentido transversal. En M. cyclopeus, la zona comprendida entre el trocánter mayor y el trocánter menor muestra un perfil más cóncavo en la cara ventral, de manera que, visto por su cara caudal, el margen ventral de la cabeza del fémur se sitúa por debajo de la zona delimitada por los dos trocánteres; en $M$. avellanarius sin embargo, el margen ventral de la cabeza del fémur se sitúa por encima.

En la epífisis distal, el cóndilo lateral de M. cyclopeus es más alto en sentido dorso-plantar que el de
M. avellanarius. El margen ventral del cóndilo lateral de M. cyclopeus forma un arco muy suave, mientras que en $M$. avellanarius el perfil es netamente circular (fig. 5G y 5H). Vistos por su cara craneal (fig. 5E y 5F), los cóndilos lateral y medial de la epífisis distal de M. cyclopeus muestran una altura similar en sentido dorso-ventral, mientras que en $M$. avellanarius los cóndilos son más asimétricos, dado que el cóndilo lateral muestra una altura dorso-ventral proporcionalmente mayor. La fosa para el músculo popliteo de $M$. cyclopeus muestra, en comparación con la de M. avellanarius, un diámetro proporcionalmente menor en relación a la superficie lateral del cóndilo. Sin embargo, la fosa de M. cyclopeus es más profunda que la de $M$. avellanarius.

Tal como ya se indicaba en el caso del húmero, de los once extremos distales conservados del fémur, nueve $(\approx 82 \%)$ están sin epifisar.

\section{Tibia}

Material. CBQ-4221, CBQ-4222 (epífisis distales izquierdas).

Descripción. La epífisis distal de M. cyclopeus es, comparada con la de M. avellanarius, proporcionalmente más estrecha en sentido transversal (figs. $5 \mathrm{~K}-5 \mathrm{~N}$ ). Vista por su cara ventral, la foseta lateral para el astrágalo de $M$. cyclopeus muestra los márgenes lateral y medial paralelos, mientras que en M. avellanarius esta misma foseta es proporcionalmente más ancha en sentido transversal, dado que el margen medial presenta un perfil algo más redondeado. Vistas por su cara caudal, las fosetas lateral y medial de $M$. cyclopeus muestran una sección redondeada, mientras que en $M$. avellanarius es más aguda (fig. 5I y 5J, tabla 2).

\section{Calcáneo}

Material. CBQ-4223, CBQ-4225, CBQ-4226, CBQ-4229，CBQ-4230，CBQ-4231，CBQ-4232 (calcáneos izquierdos), CBQ-4224, CBQ-4227, CBQ-4228, CBQ-4348 (calcáneos derechos).

Descripción. El calcáneo de M. cyclopeus es, comparado con el de $M$. avellanarius, proporcionalmente más estrecho en sentido dorso-plantar. En $M$. cyclopeus el margen dorsal de la faceta para la articulación del astrágalo sobresale menos en relación a la superficie dorsal de la mitad caudal del calcáneo. 


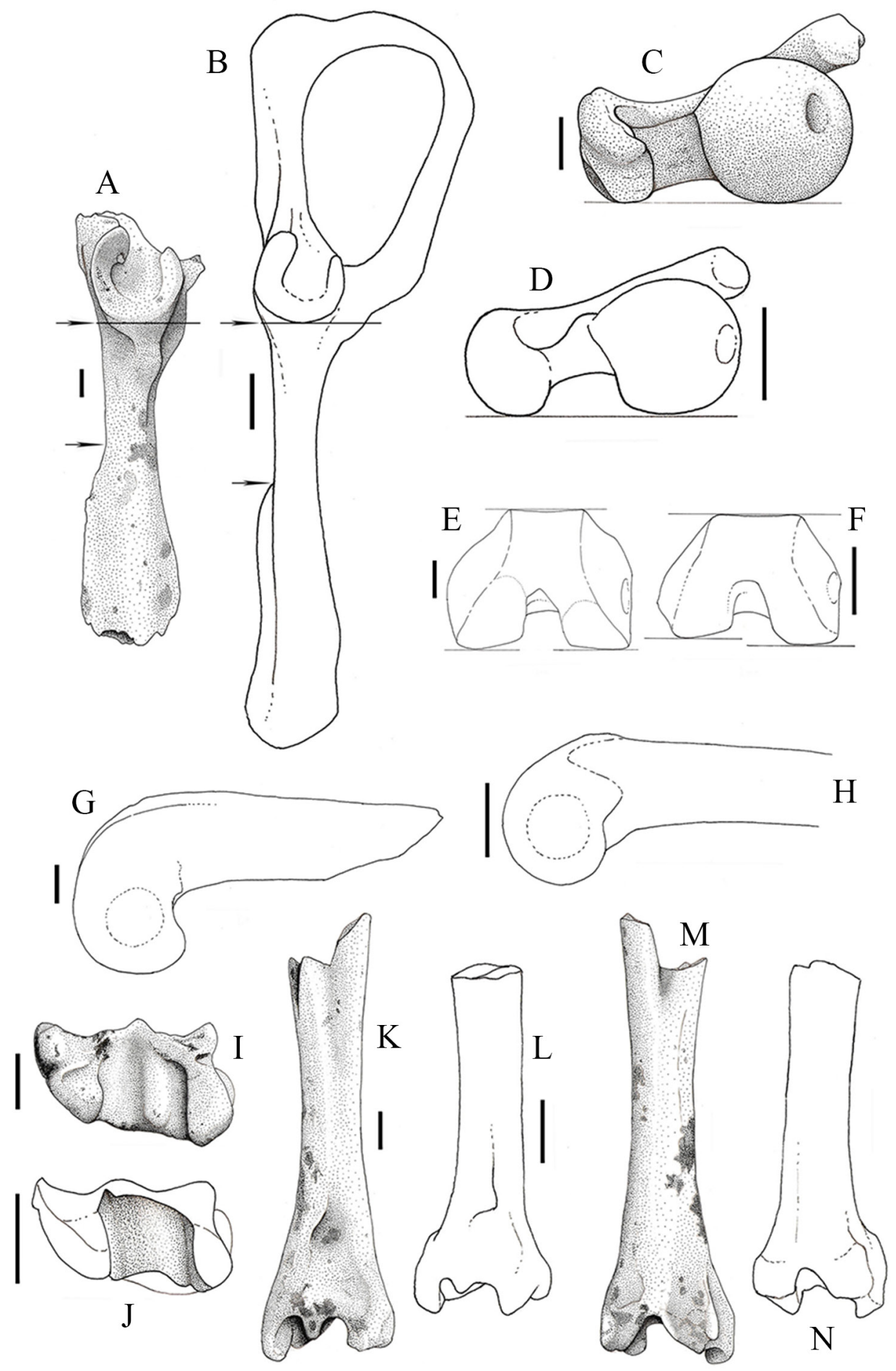

Fig. 5.-Extremidad posterior de M. cyclopeus (A: pelvis izquierda [CBQ-4220], vista lateral. C: epífisis proximal del fémur derecho [CBQ-4165], vista caudal. Epífisis distal del fémur izquierdo [CBQ-4169]: vista craneal [E] y lateral [G]. Epífisis distal de la tibia izquierda [CBQ-4222]: vista ventral [l], caudal [K] y craneal [M]). Extremidad posterior de M. avellanarius (360/93) (B: pelvis izquierda, vista lateral. D: epífisis proximal del fémur derecho, vista caudal. Epífisis distal del fémur izquierdo: vista craneal [F] y lateral $[\mathrm{H}]$. Epífisis distal de la tibia izquierda: vista ventral [J], caudal $[\mathrm{L}]$ y craneal $[\mathrm{N}])$. Escala: $1 \mathrm{~mm}$. 

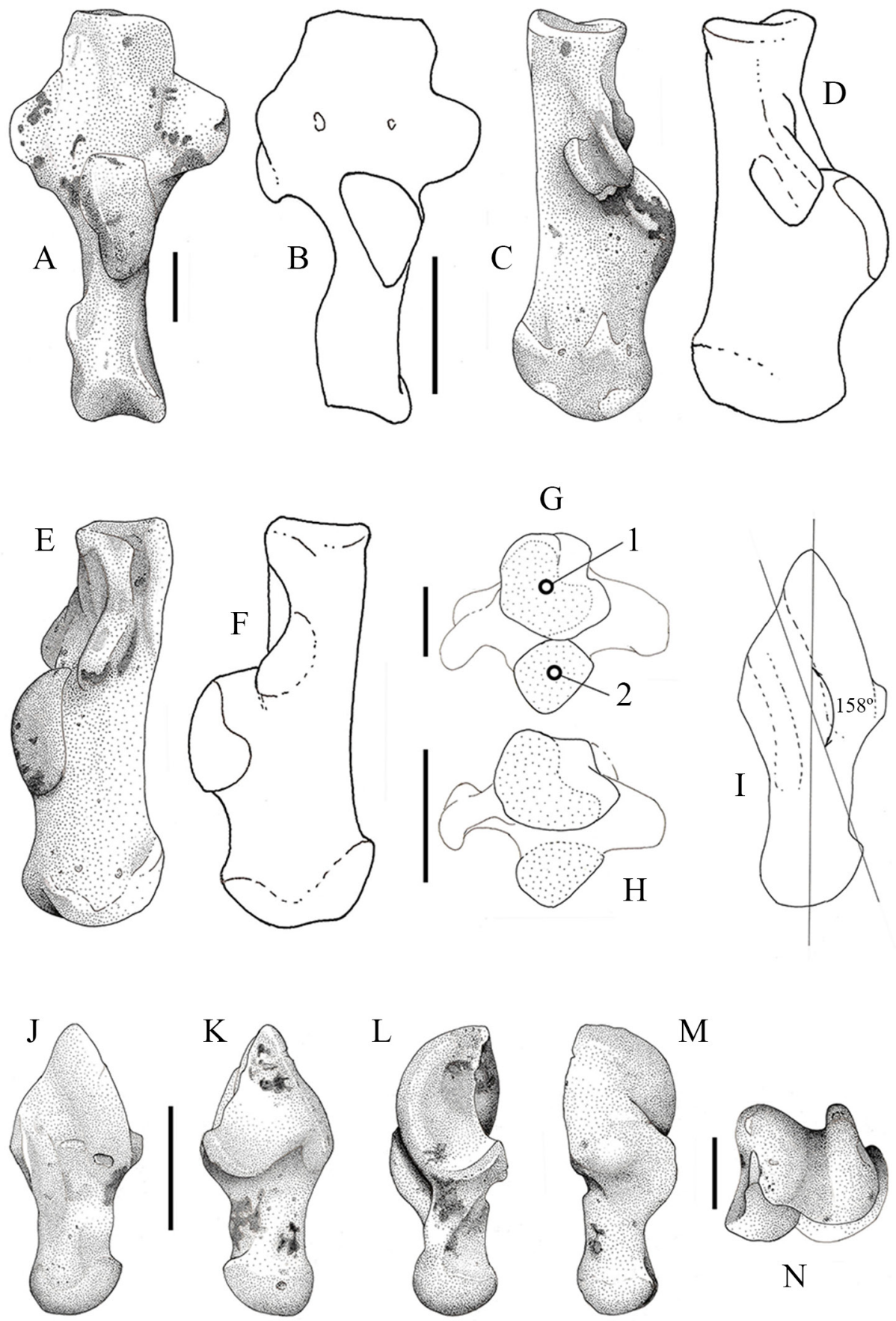

Fig. 6.-Calcáneo izquierdo de M. cyclopeus [CBQ-4229] (A: vista dorsal. C: vista lateral. E: vista medial. G: vista caudal, con las facetas para el hueso cuboideo [1] y el astrágalo [2]). Calcáneo izquierdo de M. avellanarius (360/93) (B: vista dorsal. D: vista lateral. $\mathrm{F}$ : vista medial. H: vista caudal). Astrágalo derecho de M. cyclopeus (CBQ-4353) (I: ángulo que forma el eje longitudinal con las facetas para la tibia. J: vista dorsal. K: vista ventral. L: vista medial. M: vista lateral. $\mathrm{N}$ : vista caudal). Escala: $1 \mathrm{~mm}$.

El margen lateral de esta misma faceta es ligeramente sinuoso en $M$. cyclopeus y más curvado en $M$. avellanarius (figs. 6C-6F). Dorsalmente, el margen caudal del tuber calcanei de M. cyclopeus muestra un perfil cóncavo, con los márgenes medial y lateral muy simétricos, mientras que en $M$. avellanarius 
estos son más asimétricos y la concavidad del margen caudal es marcadamente menor (fig. 6A y 6B). La faceta para el cuboides de M. cyclopeus es bilobulada, con los dos lóbulos muy simétricos; en $M$. avellanarius, sin embargo, el lóbulo plantar muestra un contorno más redondeado que el lóbulo dorsal. Vista cranealmente, la faceta para la articulación del astrágalo de $M$. cyclopeus muestra un perfil algo más agudo (fig. $6 \mathrm{G} \mathrm{y} 6 \mathrm{H}$, tabla 2 ).

\section{Astrágalo}

Material. CBQ-4234, CBQ-4351 (astrágalos izquierdos), CBQ-4353, CBQ-4233, CBQ-4349, CBQ-4350, CBQ-4352 (astrágalos derechos).

Descripción. Forma general bastante estilizada, con una relación entre la longitud y la anchura máxima de 2,21. Extremo caudal con un perfil agudo y que forma un ángulo de $63^{\circ}$. Troclea con la cresta lateral más estrecha y aguda que la cresta medial. Ambas crestas muestran un perfil transversal muy redondeado y están separadas por un surco bastante profundo. Las dos crestas forman, en relación al eje longitudinal, un ángulo de $158^{\circ}$. Vista de perfil, la cresta lateral muestra un contorno circular ligeramente alargado. Vista por su cara ventral, la faceta para el calcáneo presenta un contorno muy agudo en el margen caudal y sinuoso en el margen craneal. Esta faceta forma una superficie curvada, con un perfil en sentido transversal en forma de arco circular (figs. 6I-6N, tabla 2).

\section{Conclusiones}

La existencia de un porcentaje muy elevado de extremos proximales y distales del húmero $\mathrm{y}$ del fémur sin epifisar de M. cyclopeus se relaciona, de forma preliminar, con el alargamiento de su ciclo de vida y con el retraso en su madurez, tal como se ha constatado en el género Hypnomys (García-Martínez et al., 2011). Futuros estudios paleohistológicos permitirán confirmar o desmentir tal hipótesis.

Las principales características que diferencian M. cyclopeus de $M$. avellanarius son: 1) el sulco intertubercular de la epífisis proximal del húmero proporcionalmente más estrecho y profundo; 2) en la ulna, el olecranon es proporcionalmente más corto en sentido dorso-ventral; 3) el margen medial de la epífisis proximal del radio es más bajo en sentido dorso-ventral (fovea con una concavidad menos marcada); 4) el ilium es proporcionalmente más corto; 5) la epífisis distal del fémur es proporcionalmente más alta en sentido dorso-ventral; 6) la superficie ventral de la epífisis distal del fémur es muy aplanada; 7) los epicóndilos lateral y medial de la epífisis distal del fémur tienen una longitud dorso-ventral similar (vistos cranealmente, son muy simétricos); 8) la epífisis distal de la tibia es proporcionalmente más estrecha en sentido transversal; 9) las fosetas para el astrágalo de la epífisis distal de la tibia presentan una sección algo más redondeada en sentido transversal y 10) la mitad caudal del calcáneo es dorso-plantarmente más estrecha.

Al considerar las medidas óseas en su conjunto (tablas 1 y 2), M. cyclopeus muestra un tamaño medio un $69 \%$ superior al de $M$. avellanarius (tabla 3 ). En este sentido, cabe destacar que las diferencias de tamaño más alejadas del valor medio se relacionan con algunas de las diferencias morfológicas apuntadas anteriormente (diámetro dorso-ventral de la incisura troclear, altura del olecranon y diámetro transversal de la epífisis proximal de la ulna). Los cambios observados en el codo de M. cyclopeus, como la menor concavidad de la fóvea de la epífisis proximal del radio, la menor altura del olecranon, el diámetro dorso-ventral de la incisura troclear proporcionalmente mayor y el diámetro transversal de la epífisis proximal de la ulna proporcionalmente más ancho se podrían relacionar con cambios en el tipo de locomoción o del comportamiento, tal como sucede, por ejemplo, en los géneros Canariomys Crusafont \& Petter, 1964 e Hypnomys, mejor adaptados para la excavación que sus parientes continentales (López-Martínez \& López-Jurado, 1987; Bover et al., 2010; Quintana y Moncunill-Solé, 2014b).

Existen diferencias morfológicas significativas entre la la pelvis, el fémur, la tibia y el calcáneo de $M$. cyclopeus i M. avellanarius. Es probable que tales diferencias también queden reflejadas en el tipo de locomoción y el rango de movimientos que puede ejecutar la extremidad posterior de M. cyclopeus.

En relación a la pelvis, es frecuente observar, en los mamíferos insulares, caracterizados por una locomoción lenta y potente tipo "low gear" (Sondaar, 1977), cambios que afectan a la longitud relativa de 
Tabla 3.-Tamaño, en porcentajes, de $\boldsymbol{M}$. cyclopeus comparado con el tamaño de $\boldsymbol{M}$. avellanarius

\begin{tabular}{llll}
\hline Húmero & Ep. prox. & Diámetro dorso-ventral & $+66 \%$ \\
& & Diámetro transversal & $+59 \%$ \\
& Epífisis distal & Diámetro transversal & $+60 \%$ \\
& & Diámetro transversal sup. articulación & $+69 \%$ \\
& & Diámetro dorso-ventral sup. articulación & $+43 \%$ \\
Radio & Ep. prox. & Diámetro antero-posterior & $+70 \%$ \\
& & Diámetro antero-posterior & $+60 \%$ \\
Ulna & Epífisis proximal & Diámetro transversal & $+62 \%$ \\
& & Diámetro dorso-ventral incisura troclear & $+82 \%$ \\
& & Profundidad cráneo-caudal incisura troclear & $+63 \%$ \\
Fémur & Altura olecranon & $+44 \%$ \\
& Epífisis proximal & Diámetro transversal & $+127 \%$ \\
& & Distancia entre trocánters & $+61 \%$ \\
Tibia & Diámetro transversal caput femoris & $+76 \%$ \\
& Ep. dist. & Diámetro antero-posterior Caput femoris & $+79 \%$ \\
& & Diámetro transversal & $+75 \%$ \\
& & Diámetro antero-posterior & $+59 \%$ \\
& & Diámetro transversal & $+63 \%$ \\
& & Diámetro antero-posterior & $+85 \%$ \\
& & Diámetro transversal & $+63 \%$ \\
& &
\end{tabular}

la zona isquio-púbica e iliaca. Así, en N. rex se produce un acortamiento bastante acusado de la zona isquio-púbica (Quintana, 2005), mientras que en Myotragus balearicus Bate, 1909 el ilium es proporcionalmente más corto y la zona isquio-púbica más alargada (Alcover et al., 1981).

Al desconocer las características morfo-esqueléticas del antepasado continental de M. cyclopeus, resulta difícil saber qué diferencias corresponden a caracteres primitivos y cuáles son derivados, es decir, debidos a la evolución insular. Si bien es cierto que en $M$. cyclopeus existe un importante incremento del peso corporal en relación a $M$. avellanarius, comprendido entre un $132 \%$ y un $335 \%$ (100 g de $M$. cyclopeus frente a los 23-43 g de M. avellanarius) (Moncunill-Solé et al., 2014; Papillon et al., 2000), es probable que tal incremento no suponga, para un mamífero de talla relativamente pequeña, unos problemas biomecánicos equiparables a los existentes en otros mamíferos más grandes, como el conejo gigante de Menorca ( $N$. rex) o el bóvido endémico de las Baleares (M. balearicus). Además, la existencia de aves rapaces en los yacimientos de Punta Nati (Seguí, 1998), debió suponer, para M. cyclopeus, un estrés que impidió, hasta cierto punto, cambios alométricos significativos relacionados con la longitud funcional y diámetro sagital de los huesos largos de las extremidades y al tipo de locomoción, de manera parecida a las observaciones realizadas en Hypnomys (Quintana \& Moncunill-Solé, 2014b). Sin embargo, hasta que no se recuperen huesos largos completos de M. cyclopeus no es posible saber hasta que punto es posible establecer paralelismos entre estos dos mamíferos.

\section{AGRADECIMIENTOS}

El autor está especialmente agradecido al Dr. Andrew Kitchener (Principal Curator of Vertebrates, National Museums of Scotland, Department of Natural Sciences) por el préstamo temporal de los dos esqueletos de $M$. avellanarius, y a Majo León (Directora del Museo Municipal del Bastió de Sa Font, Ciutadella de Menorca), por ejercer de mediadora entre el Dr. Kitchener y el autor. Al Dr. David Martínez-Alba (Institut Catalá de Paleontología Miquel Crusafont, Barcelona) por la revisión del resumen en inglés. Las sugerencias y correcciones del Dr. Jordi Agustí Ballester (Institut Català de Paleoecologia Humana i Evolució Social, Tarragona) y del Dr. Francisco Javier Ruiz Sánchez (Instituto de Investigaciones Científicas y Tecnológicas, Ecuador) han ayudado a mejorar el manuscrito original. La realización de este trabajo ha sido posible gracias a la ayuda del Ministerio de Economia y Competitividad, Gobierno de España (I+D MINECO CGL2012-34459). 


\section{Referencias}

Agustí, J.; Moyà-Solà, S. \& Pons-Moyà, J. (1982). Une espèce géante de Muscardinus Kaup, 1829 (Gliridae, Rodentia, Mammalia) dans le gisement karstique de Cala es Pou (Miocène supérieur de Minorque, Baléares). Geobios, 15: 783-789. http://dx.doi.org/ 10.1016/S0016-6995(82)80008-9.

Alcover, J.A.; Moyà-Solà, S. \& Pons-Moya, J. (1981). Les quimeres del passat. Els vertebrats fòssils del PlioQuaternari de les Balears i Pitiüses. Monografies Científiques 1. Editorial Moll, Palma de Mallorca, 260 pp.

Bover, P.; Alcover, J.A.; Michaux, J.J.; Hautier, L. \& Hutterer, R. (2010). Body shape and life style of the extinct balearic dormouse Hypnomys (Rodentia, Gliridae): new evidence from the study of associated skeletons. PLoS ONE, 5: 1-11. http://dx.doi.org/ 10.1371/journal.pone.0015817.

García-Martínez, R.; Marín-Moratalla, N.; Jordana, X. \& Köhler, M. (2011). First results from bone histology of the giant fossil dormouse Hypnomys. In: Book of abstracts of the First International Symposium on Paleohistology (Köhler, M.; Jordana, X. \& MarínMoratalla, N.; Eds.). Institut Català de Paleontología Miquel Crusafont, Sabadell (Barcelona), 78.

López-Martínez, N. \& López-Jurado, L.F. (1987). Un nuevo múrido gigante del Cuaternario de Gran Canaria Canariomys tamarani nov. sp. (Rodentia, Mammalia). Interpretación filogenética y biogeográfica. Doñana, 2: 1-66.

Moncunill-Solé, B.; Jordana, X.; Marín-Moratalla, N.; Moyà-Solà, S. \& Köhler, M. (2014). How large are the extinct giant insular rodents? New body mass estimations from teeth and bones. Integrative Zoology, 9: 197-212. http://dx.doi.org/10.1111/1749-4877.12063.

Paillon, Y.; Butet, A.; Paillat, G. \& Milan-Pena, N. (2000). Insectivores et Rongeurs de France: le muscardin,
Muscardinus avellanarius (Linné, 1758). Arvicola, 12(2): 39-51.

Quintana, J. (1998). Aproximación a los yacimientos de vertebrados del Mio-Pleistoceno de la isla de Menorca. Bolletí de la Societat d'Història Natural de les Balears, 41: 101-117.

Quintana, J. (2005). Estudio morfológico y funcional de Nuralagus rex (Mammalia, Lagomorpha, leporidae). Tesis Doctoral, Universidad Autónoma de Barcelona, $360 \mathrm{pp}$.

Quintana, J. \& Moncunill-Solé, B. (2014a). Hypolagus balearicus Quintana et al., 2010 (Mammalia: Leporidae): new data from the Neogene of Eivissa (Balearic Islands: Western Mediterranean). Geodiversitas, 36(2): 283-310. http://dx.doi.org/10.5252/g2014n2a4.

Quintana, J. \& Moncunill-Solé, B. (2014b). Reconsidering locomotor habits and life style of the balearic insular giant rodent Hypnomys Bate, 1918 from the allometry of the limb long bones. Comptes Rendus Palevol, 13(4): 297-306. http://dx.doi.org/10.1016/j. crpv.2013.11.003.

Seguí, B. (1998). Succesió estratigràfica d'aus en els rebliments càrstics de les Gymnèsies. Tesis doctoral, Universitat de les Illes Balears, 194 pp.

Sevilla, P.; Quintana, J. \& Furió, M. (2013). Bats in islands: new data from the Pliocene-Early Pleistocene fossils from Menorca (Spain). XXIX Jornadas de la Sociedad Española de Paleontología: la paleontología del Paleozoico. Simposio del proyecto PICG 596. Centro Paleobotánico, Real Jardin Botánico de Córdoba, Universidad Complutense de Madrid y Sociedad Española de Paleontología, 187-188.

Sondaar, P.Y. (1977). Insularity and its effect on mammal evolution. In: Major patterns in vertebrate evolution (Hecht, M.K.; Goody, P.C. \& Hecht, B.M., Eds.). Plenum Publishing Corporation, 671-707. http://dx. doi.org/10.1007/978-1-4684-8851-7_23. 